\title{
Improving Completeness of Inpatient Medical Records in Menelik II Referral Hospital, Addis Ababa, Ethiopia
}

\author{
Kasu Tola, ${ }^{1}$ Haftom Abebe, ${ }^{1}$ Yemane Gebremariam, ${ }^{1}$ and Birhanu Jikamo ${ }^{2}$ \\ ${ }^{1}$ Mekelle University College of Health Sciences School of Public Health, Mekelle, Ethiopia \\ ${ }^{2}$ Hawassa University College of Medicine and Health Sciences School of Public and Environmental Health, Hawassa, Ethiopia \\ Correspondence should be addressed to Kasu Tola; kasu.tola@yahoo.com
}

Received 10 December 2016; Revised 3 March 2017; Accepted 28 March 2017; Published 12 April 2017

Academic Editor: Ronald J. Prineas

Copyright (C) 2017 Kasu Tola et al. This is an open access article distributed under the Creative Commons Attribution License, which permits unrestricted use, distribution, and reproduction in any medium, provided the original work is properly cited.

\begin{abstract}
Introduction. The incompleteness of medical records is a significant problem that affects the quality of health care services in many hospitals of Ethiopia. Improving the completeness of patient's records is an important step towards improving the quality of healthcare. Methods. Pre- and postintervention study was conducted to assess improvement of inpatient medical record completeness in Menelik II Referral Hospital from September 2015 to April 2016. Simple random sampling technique was used. Data was collected using data extraction checklist and independent sample $t$-test was used to compare statistical difference that exists between pre- and postintervention outcomes at confidence interval of $95 \%$ and $P$ value less than 0.05 was considered statistically significant. Result. The overall inpatient medical record completeness was found to be $84 \%$ after intervention. An enhancement of completeness and reporting of inpatient medical record completeness increased significantly from the baseline $73 \%$ to $84 \%$ during postintervention evaluation at $P$ value $<0.05$. Conclusion and Recommendation. The finding of this project suggests that a simple set of interventions comprising inpatient medical record format and training healthcare provider showed a significant improvement in inpatient medical record completeness. The Quality Officer and Chief Executive Officer of the study hospital are recommended to design and launch intervention programs to improve medical record completeness.
\end{abstract}

\section{Introduction}

Medical record completeness is a key performance indicator that is related with delivery of healthcare services in the hospital [1]. At hospital level, statistics collected from medical records are used to review the incidence and type of diseases treated and different procedures performed. At hospital level statistics derived from the daily bed census and medical records are used to assess the utilization of services and enable the hospital to make appropriate financial and administrative plans and to conduct vital research [2].

Patient medical record review is the most applied technique to investigate adverse events in hospitals. The determination with which information is recorded may influence the visibility of adverse events. Poor quality of the information in patient medical records may be a cause or a consequence of poor quality of care and associated with higher rates of adverse events [3]. Better quality of healthcare data in patient medical records can affect clinical and administrative decision making in health economics and patient safety [4].

Adverse events occur in an estimate of 2.9 to 3.7 percent of acute care hospitalizations in the United States of America (USA) and it is estimated that between 44,000 and 98,000 patients die in hospitals each year as a result of medical error explained as the failure of planed action to be completed as intended [5].

Despite the importance of medical records to high quality and efficient care management of patients' medical records, especially in developing countries like Ethiopia, it has not been a priority, generally inadequately supported and poorly managed. The study done in a rural hospital in Ethiopia shows that only $45.7 \%$ of medical records were complete [6].

A facility based cross-sectional study was conducted in Ayder Referral Hospital and six-month data have been assessed and showed that $36.7 \%$ was inaccurate [7]. 
The study done in a Dalefage Primary Hospital, West Afar, Ethiopia, showed that an enhancement of completeness and reporting of inpatient medical record completeness improved significantly from the baseline $0 \%$ to $73.6 \%$ during postintervention evaluation [8].

In Menelik II Referral Hospital baseline assessments were collected and inpatient medical record completeness showed $73 \%$ which is low against the standard in which medical record completeness is expected to be $100 \%$. In line with this there is a gap of study on medical record completeness particularly inpatient medical records. Knowledge gap and shortage of medical record format were accepted as root cause for existence of incomplete inpatient medical records.

Objective of the study is to improve the completeness of inpatient medical records from $73 \%$ to $93 \%$ at the end of April 30, 2016, at Menelik II Referral Hospital.

A patient medical record provides two important functions; the first helps to support direct patient care by assisting physician on clinical decision making and provides communication. The second provides a legal record of care given and helps as a source of data to support clinical audit, research, resource allocation, monitoring and evaluation, epidemiology, and service planning [9-11].

Improving medical record completeness services is an important step towards improving the quality of healthcare. It can also provide valuable information to help measure progress and effectiveness. The medical record has become an important legal document; good medical records are essential not only for the present and future care of the patient but also as a legal document to protect the patient and the hospitals from litigation $[1,2]$.

Medical record is a very important document that is used to communicate and document critical information among health professionals. The incompleteness of medical records compromises the quality of care of patient's and results in different medical errors and patient dissatisfaction. To alleviate the problem the medical record completeness is part of national key performance indicators to monitor the magnitude of the problem and intervene according to the necessity.

\section{Methodology}

2.1. Setting/Study Area. The study was conducted at Menelik II Referral Hospital, governmental hospital found in capital city of Ethiopia in Addis Ababa. Menelik II Referral Hospital has various professionals that included 59 physicians, 203 nurses, 123 other health professionals, and 250 administrative staff, making a total of 635 staff. The study was conducted from September 2015 to April 2016.

2.2. Study Design. Pre- and postintervention study was conducted at inpatient departments of Menelik II Referral Hospital.

2.3. Population. All inpatient medical records of patients treated and discharged from Menelik II Referral Hospital were sourced for review medical records after implementing the best intervention. Baseline data were collected in September 2015 and postintervention data was collected in May 2016 and schedule for intervention was carried out in January, February, March, and April 2016.

2.4. Sample Size Determination. The sample size calculation for comparing proportions was used to make a valid statistical computation and conclusion; the following sample size calculation for comparing two proportions was made [12]:

$$
n=\frac{(Z \alpha / 2+Z \beta)^{2} P(1-P)}{(P-P o)^{2}}
$$

where $n$ is sample size and $P=(P o+P 1) / 2, P o=73 \%=$ 0.73 (baseline data surveys were collected and analysis was done which gives $73 \%$ of inpatient medical record as being completed in Menelik II Referral Hospital). $P 1=93 \%=0.93$ (planned proportion after the best intervention implemented in Menelik II Referral Hospital is expected to be $93 \%$ because of shortage of resources and time to accomplish the tasks). $P=P o+P 1 / 2=0.83$ (average population proportion that is between preintervention and postintervention proportion); $Z \alpha / 2$ with $95 \%$ confidence interval equal to 1.96 , power $=$ $80 \%$, and $Z \beta=0.84$.

$$
\begin{aligned}
& n=\frac{(1.96+0.84)^{2} 0.83(1-0.83)}{(0.83-0.73)^{2}} \\
& n=111 .
\end{aligned}
$$

2.5. Sampling Technique and Procedures. Simple random sampling technique was used during the study period which means that for preintervention, September 2015, the total discharged patients were 605 and among these 50 medical records were sampled and for postintervention, May 2016, the total discharged patients were 582 and among these 111 medical records were sampled, respectively, using lottery methods. The above sample size was taken based on Ethiopian Federal Ministry of Health Hospital performance and monitoring improvement reports [13].

\subsection{Intervention}

2.6.1. Training for Inpatient Healthcare Worker (Physician and Nurse). Training for physician and nursing staff consists of the following:

(i) Awareness and sensitization creation on the importance of medical records.

(ii) Medical record as part of hospital reform.

(iii) Medical record as part of hospital key performance indicator for quality of care.

2.6.2. Avail Medical Record Format. During intervention implementation the main focus was to provide training for inpatient healthcare worker (physician and nurses) for one day for 122 nurses and 67 physicians totally for 189 healthcare providers on inpatient medical record completeness as well 
hospital reform by providing onsite training to solve lack of awareness and knowledge gap on the overall hospital reform.

2.7. Evaluation. The type of evaluation that was conducted is the cycle of problem solving, PDSA cycle of continuous quality improvement.

\section{Process indicators}

(i) Availability of necessary formats

(ii) Trained healthcare provider (Physician and Nurse)

Outcome indicators

(i) Score of completeness of inpatient medical records after intervention.

2.8. Data Collection Procedure and Quality Control. The training was given to two nurses for one day. These trained data collectors used data extraction checklist and collect information from medical charts. To maintain data quality, training was given to data collectors and supervision was carried out by principal investigator on daily basis to check completeness and consistency so as to ensure quality of data, during the data collection procedures.

\subsection{Operational Definition}

Medical Record. They are papers that document the care and treatment a patient received.

Completeness of Medical Record. It is the presence of all the necessary information of patients based on the standard formats attached at the annex and all entries are dated and signed.

Inpatient Medical Record. It is the official record of patient that contains information of admitted patients to general ward.

2.10. Data Entry and Analysis Procedure. After data is collected, it was coded and entered into Epi data of version 3.1 and was exported to SPSS for windows version 22 for cleaning, editing, and analysis, and $t$-test was used to compare statistical difference that exists between preintervention and postintervention. $P$ value less than 0.05 was considered statistically significant. Ethical clearance was obtained from Mekelle University College of Health Science, School of Public Health, as the study was conducted as part of Master's thesis for the first author and prior to commencement of the study. Official letter of permission from the school was submitted to Menelik II Referral Hospital administration in order to conduct the project. Following this, searching and obtaining of the selected samples' medical record was processed with assigned person. Finally, strict care for the patients' medical records and the confidentiality of records that could identify study participants was protected.

\section{Result}

The completeness of medical records was assessed in terms of physician note, physician order sheet, nursing care plan, medication administration sheet, and discharge summary. Accordingly, the result showed that physician note format was attached for 111 (100\%) and completed for 103 (92.8\%), physician order sheet was attached for $111(100 \%)$ and completed for 107 (96.4\%), nursing care plan was attached for 109 (98.2\%) and completed for 85 (76.6\%), medication administration format was attached for 103 (92.8\%) and completed for 78 $(70.3 \%)$, and at last discharge summary was attached for 107 (96.4\%) and completed for 93 (83.8\%) (Figure 1).

There are prepared standardized formats and three of them were completed by physician (inpatient physician notes, physician order sheet, and discharge summary) and two of them completed by nurses (nursing care plan and medication administration sheet). An enhancement of completeness and reporting of inpatient medical record completeness improved significantly from the baseline $73 \%$ before intervention to $84 \%$ after intervention $(P$ value $<0.05$, Table 1$)$.

Since calculated $t$-value exceeds the critical value, the null hypothesis has been rejected and the alternative hypothesis has been accepted, implying that intervention done has brought a significant change.

\section{Discussion}

The result of the study showed that the intervention done has increased the overall inpatient medical record completeness by $11 \%$ from $73 \%$ to $84 \%$ ( $P$ value $<0.05)$. When compared with study done in Netherland the nursing record was unavailable in $1 \%$ of the patient records and the medication administration list in $21 \%$ of the reviewed patient records but relatively similar to the study in which medication administration list is incomplete for $29.7 \%$ [3].

When compared with aspects of medication administration sheet the study done in England shows that the medication history in the hospital medical record is often incomplete, as $26 \%$ of used medication is not recorded. Similarly in this study medication administration sheet is incomplete for $29.7 \%$ of the medical records [14].

Aspects of discharge summary in this study: $16.2 \%$ of inpatient medical record is incomplete in comparison with study done in Canada which shows discharge summaries were assessed for completeness and accuracy. Most items were completely reported with given items missing in $5 \%$ of summaries. However there is improvement of completeness of discharge summary as compared to preintervention but low as compared to study done in Canada. The reason for this observation might be that the country gives due attention for medical records for better health information and decision making [15].

The study done in a rural hospital in Ethiopia shows that the proportion of medical records that were complete increased significantly (6.5\% preintervention and $45.7 \%$ postintervention, $P<0.01)$; in line with this, in our study there is also significant improvement in inpatient medical record completeness which implies giving due attention to 


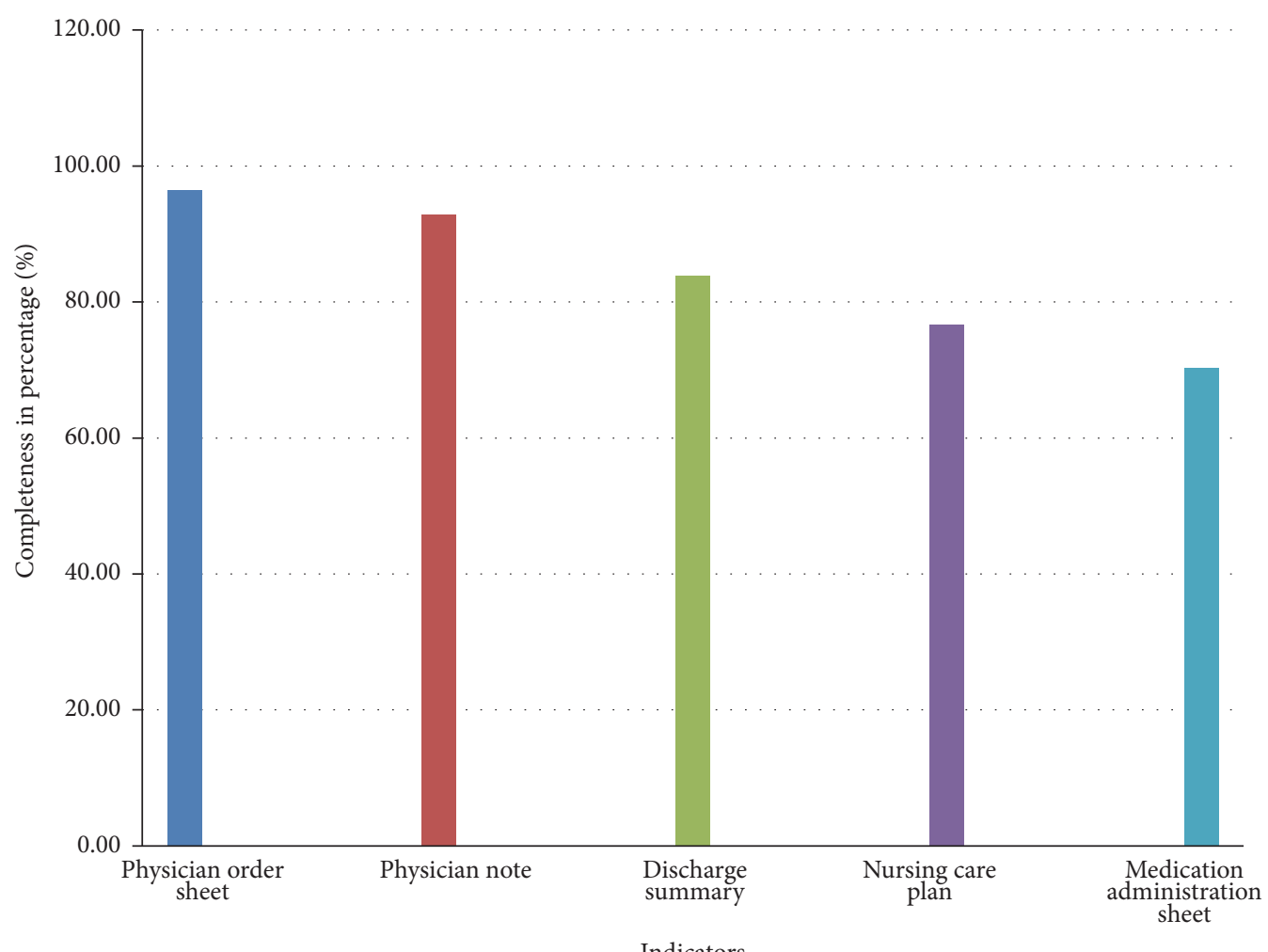

FIgURE 1: Status of completeness of medical record completeness in Menelik II Referral Hospital, Addis Ababa, Ethiopia, May 2016.

TABLE 1: Pre- and postintervention changes in medical record completeness in Menelik II Referral Hospital, Addis Ababa, Ethiopia, May 2016.

\begin{tabular}{lcc}
\hline Indicators & Preintervention, September 2015 & Postintervention, May 2016 \\
\hline Inpatient physician note & $96 \%$ & $92.8 \%$ \\
Physician order sheet & $96 \%$ & $96.4 \%$ \\
Nursing care plan & $70 \%$ & $76.6 \%$ \\
Medication administration sheet & $40 \%$ & $70.3 \%$ \\
Discharge summary & $64 \%$ & $83.8 \%$ \\
Total & $73 \%$ & $84 \%$ \\
\hline
\end{tabular}

medical records and applying simple set of intervention can bring changes [6].

The study done in a Dalefage Primary Hospital, West Afar, Ethiopia, shows that an enhancement of completeness and reporting of inpatient medical record completeness improved significantly from the baseline $0 \%$ to $73.6 \%$ during postintervention evaluation. Similar to our study after introduction of simple intervention inpatient medical record completeness improves from the baseline $73 \%$ to $84 \%$ during postintervention evaluation. This implies that, by implementing a set of intervention, it can bring improvement in completeness of medical records [8].

\section{Conclusion}

The overall inpatient medical record completeness in Menelik II Referral Hospital was $84 \%$ and the higher rate of completeness was seen in inpatient physician order sheet $96.4 \%$ completed whereas the least completed was inpatient medication administration sheet $70.3 \%$ completed. The finding of this project suggests that a simple set of intervention availing inpatient medical record format and training healthcare provider improves the inpatient medical record completeness. This project indicates that applying strategic problem solving to medical record completeness can be effective in improving quality of healthcare.

\section{Recommendations}

(1) It is better if the Health Management Information System Department takes special consideration on full implementation and proper management of inpatient medical records.

(2) Intensive and continuous training should be given for the healthcare provider by responsible body. 
(3) The Quality Officer and Chief Executive Officer at the administrative positions of the study hospital are recommended to design and launch intervention programs to improve medical record completeness.

(4) Effective long-term follow-up is needed to assess the sustainability of intervention by inpatients department head.

(5) Quality improvement project of this approach should be encouraged to be applied in other departments of hospital to enhance quality of healthcare services by quality team.

\section{Conflicts of Interest}

All authors declare that they have no competing financial or any other interest in relation to the work.

\section{Authors' Contributions}

Kasu Tola conceived the study, undertook statistical analysis, and drafted the paper. Dr. Haftom Temesgen, Yemane Gebremariam, and Birhanu Jikamo made major contributions to the study design and statistical analysis. All authors contributed to the writing of the paper and approved its submitted version.

\section{Acknowledgments}

The authors appreciate the management of Menelik II Referral Hospital and Healthcare provider working in inpatient departments. Kasu Tola also extends his thanks to Dr. Haftom Temesgen, Mr. Yemane Gebremariam, Mr. Dawit Tatek, and Mr. Birhanu Jikamo for their inspiration and technical support in many ways.

\section{References}

[1] M. Helfand and M. Freeman, Evidence-Based Synthesis Program Assessment and Management of Acute Pain in Adult Medical Inpatients: Portland VA Health Care System, Oregon EvidenceBased Center: A Systematic Review, 2008.

[2] WHO, Medical Records Manual: A Guide For Developing Countries Revised and Updated, 2006.

[3] M. Zegers, M. C. De Bruijne, P. Spreeuwenberg, C. Wagner, P. P. Groenewegen, and G. Van Der Wal, "Quality of patient record keeping: an indicator of the quality of care?" BMJ Quality and Safety, vol. 20, no. 4, pp. 314-318, 2011.

[4] I. T. Adeleke, A. O. Adekanye, K. A. Onawola et al., "Data quality assessment in healthcare: a 365-day chart review of inpatients' health records at a Nigerian tertiary hospital," Journal of the American Medical Informatics Association, vol. 19, no. 6, pp. 1039-1042, 2012.

[5] AHRQ, "Making health care safer II: an updated critical analysis of the evidence for patient safety practices," AHRQ Evidence Report 211, 2013.

[6] R. Wong and E. H. Bradley, "Developing patient registration and medical records management system in Ethiopia," International Journal for Quality in Health Care, vol. 21, no. 4, pp. 253-258, 2009.
[7] K. Tadesse, E. Gebeye, and G. Tadesse, "Assessment of health management information system implementation in Ayder referral hospital, Mekelle, Ethiopia," International Journal of Intelligent Information Systems, vol. 3, no. 4, pp. 34-39, 2014.

[8] N. M. Dima, Improving the completeness of medical records at inpatient department of Dalefage Primary Hospital, west Afar, Ethiopia [Masters of Hospital and Health Care Administration], School of Graduate Studies of Addis Ababa University, 2014.

[9] N. Tavakoli, M. Jahanbakhsh, M. Akbari, and M. Baktashian, "The study of inpatient medical records on hospital deductions: an interventional study," Journal of Education and Health Promotion, vol. 4, article 38, 2015.

[10] AHRQ, "Enabling patient-centered care through health information technology," U.S. Department of Health and Human Services Report, 2012.

[11] P. C. Tang, M. P. Larosa, and S. M. Gorden, "Use of computerbased records, completeness of documentation, and appropriateness of documented clinical decisions," Journal of the American Medical Informatics Association, vol. 6, no. 3, pp. 245251, 1999.

[12] H. Wang, "Sample size calculation for comparing proportions," in Wiley Encyclopedia of Clinical Trials, pp. 1-11, John Wiley \& Sons, 2007.

[13] MOH Federal Democratic Republic of Ethiopia, Hospital Performance Monitoring and Improvement Manual, 2011.

[14] K. Legault, J. Ostro, Z. Khalid, P. Wasi, and J. J. You, “Quality of discharge summaries prepared by first year internal medicine residents," BMC Medical Education, vol. 12, no. 1, article 77, 2012.

[15] M. Greiver, J. Barnsley, R. H. Glazier, B. J. Harvey, and R. Moineddin, "Measuring data reliability for preventive services in electronic medical records," BMC Health Services Research, vol. 12, no. 1, article 116, 2012. 


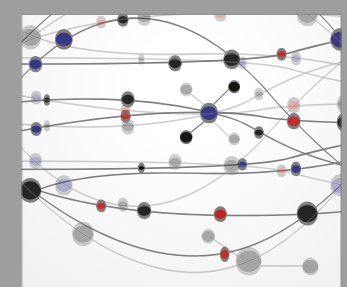

The Scientific World Journal
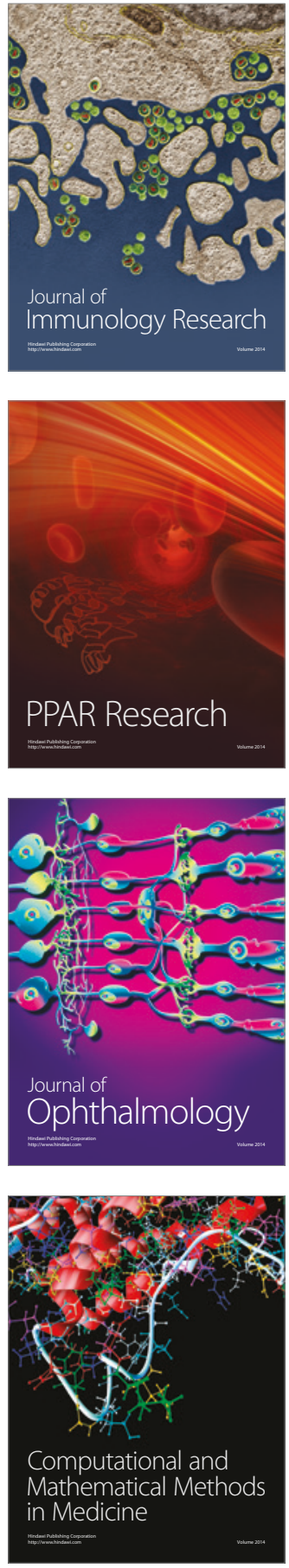

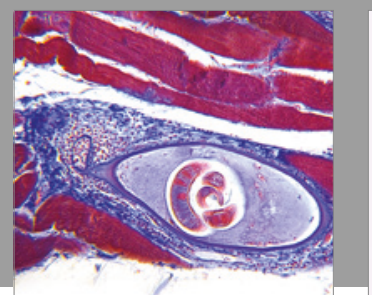

Gastroenterology Research and Practice
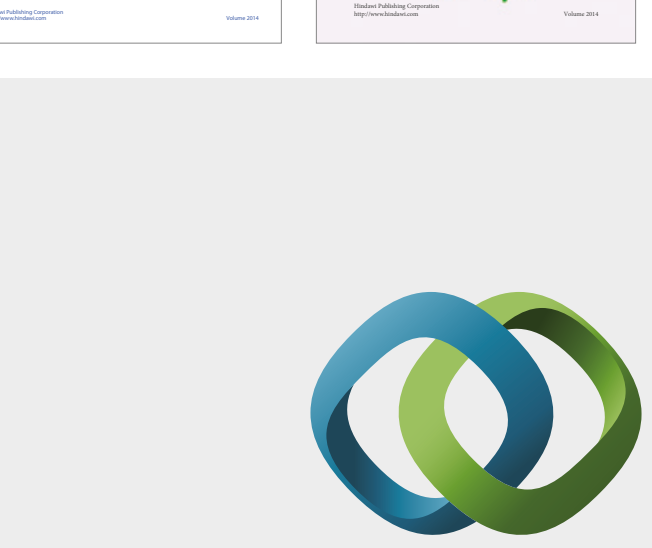

\section{Hindawi}

Submit your manuscripts at

https://www.hindawi.com
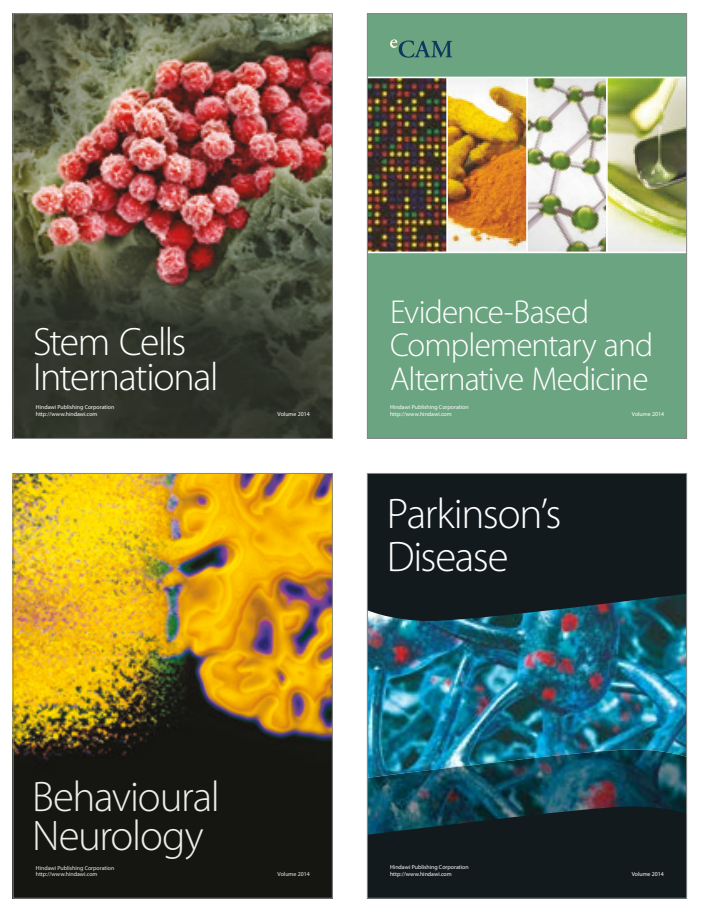


Disease Markers
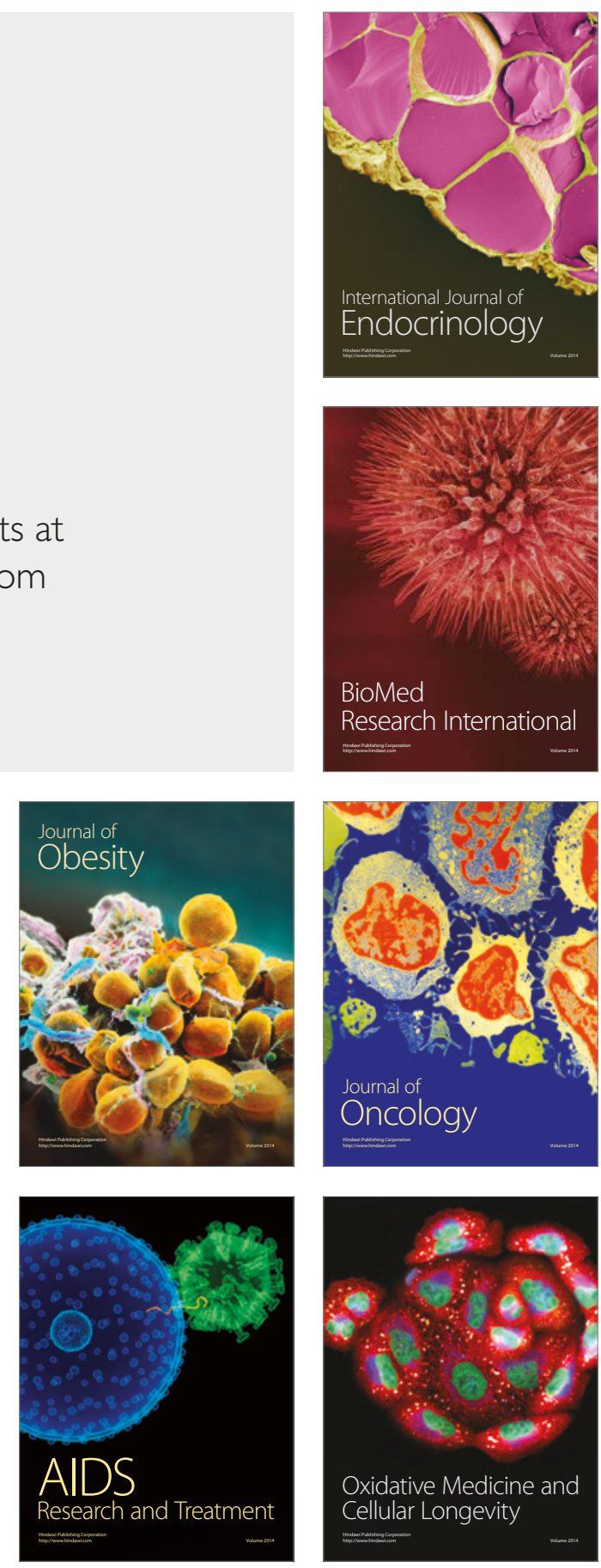